\title{
Verification of virtual reality to evaluate deficiencies in cognitive function among patients with schizophrenia in the remission stage: a cross- sectional study
}

\section{Manli Huang}

Zhejiang University School of Medicine First Affiliated Hospital

Shangda Li

Zhejiang University School of Medicine First Affiliated Hospital

\section{Bing Sun}

Ningbo Psychiatric Hospital

\section{Hailong Lyu}

Zhejiang University School of Medicine First Affiliated Hospital

\section{Weijuan Xu}

Zhejiang University School of Medicine First Affiliated Hospital Jianping Jiao

Zhejiang University School of Medicine First Affiliated Hospital

\section{Fen Pan}

Zhejiang University School of Medicine First Affiliated Hospital

\section{Jianbo Hu}

Zhejiang University School of Medicine First Affiliated Hospital

Jinkai Chen

Zhejiang University School of Medicine First Affiliated Hospital

\section{Yaping Chen}

Ningbo Psychiatric Hospital

Xu Yi ( $\nabla$ xuyizju@zju.edu.cn )

Zhejiang University School of Medicine First Affiliated Hospital https://orcid.org/0000-0002-8773-8264

\section{Research article}

Keywords: virtual reality; schizophrenia; MCCB; Cognitive function

Posted Date: August 19th, 2020

DOI: https://doi.org/10.21203/rs.3.rs-57436/v1 
License: (c) (i) This work is licensed under a Creative Commons Attribution 4.0 International License. Read Full License 


\section{Abstract}

Background: Schizophrenia is associated with widespread cognitive impairments. The MATRICS Consensus Cognitive Battery (MCCB) is most frequently used to assess cognitive function. However, the MCCB test can be time consuming for the clinician. Virtual reality (VR) has emerged as an adjunctive tool to overcome this limitation, which provides a new means of assessing cognitive function.

Methods: The study is to explore the validity and safety of using VR technology to assess cognitive function in Han Chinese patients with schizophrenia. The virtual reality supermarket training system (VRSTS), which simulates real-life supermarkets, was used to assess cognitive function. Thirty-two patients with schizophrenia (SZs) and 25 healthy controls (HCs) received the VRSTS and MCCB assessments. A diagnosis model was created based on the outcomes of VRSTS to classify SZs and HCs by cognitive impairment.

Results: Significant differences in completion time between the SZs and HCs were detected on the VRSTS. SZs spent more time completing tasks than HCs. The outcome of VRSTS was significantly correlated with the MCCB. Moreover, the diagnosis model has a sensitivity of $88.89 \%$ and a specificity of $88.89 \%$.

Conclusions: These results provide support for VR technology in the assessment of cognitive impairment in schizophrenia among Han Chinese patients.

Trial registration: China Clinical Trial Registry, ChiVTR1800016121. Registered 13 May 2018, http://www.chictr.org.cn/showproj.aspx?proj=27233

\section{Background}

Schizophrenia is a complex, heterogeneous behavioral and cognitive syndrome characterized by positive symptoms, negative symptoms and cognitive impairment [1, 2]. Patients with schizophrenia (SZs) exhibit damaged cognitive function, including reduced attention and memory, and difficulties with executive functioning[3]. The MATRICS Consensus Cognitive Battery (MCCB), which includes 10 different cognitive subtests, is an accepted standard for measuring cognitive change in schizophrenia and has been recommended by the United States Food and Drug Administration (FDA) to assess cognitive impairment in schizophrenia $[4,5]$. It demonstrates excellent reliability and practicality. Recently, studies have shown that the MCCB is both applicable for first-episode schizophrenia and chronic schizophrenia [6]and investigations using the MCCB have focused on different cognitive domains in patients with schizophrenia. A study showed that SZs' parents have conspicuous dysfunction in domains of working memory, problem reasoning and visual learning compared with HCs' parents[7]. However, the MCCB requires a well-trained psychiatrist, and it takes approximately one hour to evaluate cognitive function. Moreover, the MCCB sometimes is complicated for some patients with schizophrenia. And some patients may feel bored and exhausted to a point where they cannot complete the assessment. Thus, it is necessary to find an easier and more attractive method to evaluate cognitive function. 
Virtual reality (VR) has emerged as a tool to overcome this limitation of the MCCB, providing a new means of assessing cognitive function. VR is a powerful tool that creates interactive computer-generated worlds that produces a sensation of being in life-sized environments[8]. It has already been used in psychiatry. The present evidence suggests that the use of virtual reality among patients with schizophrenia has great advantages. Some studies have focused on assessing cognitive function using VR technology. A virtual reality navigation task (VRNT) study reported that schizophrenia patients were significantly impaired on memory compared with normal subjects[9]. Thirty-nine patients with schizophrenia and 21 healthy comparison subjects experienced a virtual maze, and the SZs exhibited a higher rate of error [10]. Another study showed that patients with schizophrenia were deficient in life activities, medication management skills, and virtual characters recognition [11-13].

Although studies have reported the application of VR in psychiatry, there is limited literature focusing on VR applied to Han Chinese people, a study in Hong Kong showed VR was a sensitive assessment of prospective memory deficits in people with schizophrenia[14], but the efficiency and safety of VR technology are still not clear enough. Furthermore, the association between the VR method and the MCCB to assess the cognitive function of SZs lacks evidence. To date, two studies in the USA have demonstrated that the completion time of the VR task to assess functional capacity was correlated with the MCCB composite scores $[15,16]$.

The present study explored the performance of VR technology in assessing cognitive function and the reliability of distinguishing $\mathrm{HCs}$ and SZs of Han Chinese descent by cognitive function.

\section{Methods}

\section{Participants}

Thirty-five patients with schizophrenia in the Ningbo Psychiatric Hospital were recruited under the supervision of Dr. Sun Bin and 25 healthy control (HCs) volunteers, were recruited from society under the supervision of Dr. Li Shangda. All the participants entered the virtual reality environment, named virtual reality supermarket training system (VRSTS) and received the MCCB test. The outcomes of VRSTS in two groups were compared and a diagnosis model was created based on the outcomes of VRSTS to classify SZs and HCs using a method named support vector machine (SVM). Another nine SZs and 9 HCs were recruited for verification of the diagnosis model

The inclusion criteria were as follows: between 18 and 55 years old; met the ICD-10 criteria for schizophrenia; on remission stage: Positive and Negative Syndrome Scale item scores of $\leq 3$ or SAPS and SANS item scores of $\leq 2$ for at least 6 months according to Andreasen's criteria[17]; only received atypical antipsychotics; normal vision and right-handedness. Exclusion criteria: a history of brain trauma, epilepsy and other neurological diseases or serious physical diseases; a diagnosis of a history of mental retardation and a history of substance abuse in the past 30 days (except smoking); received electroconvulsive therapy in the past year; a history of using typical antipsychotics; and pregnant women or those who planned to get pregnant. 
Three people with schizophrenia were declined because they cannot understand the instructions of MCCB so that they cannot complete it. All SZs and HCs were matched by gender and age.

After description of the study to the subjects, written informed consent was obtained before the study were conducted and were offered an incentive of $\$ 50$ per session. The study was approved by the ethics committee of the First Affiliated Hospital of the Medical School of Zhejiang University, in accordance with the Helsinki Declaration (No. 2018533) and was already registered in China Clinical Trial Registry and registration number is No.ChiVTR1800016121

\section{Virtual Reality Procedure}

VRSTS was designed to assess cognitive function, performing different shopping tasks with different lists. The VRSTS simulates a supermarket with a variety of shopping goods such as drinks, tea sets, kitchen ware, fruits and vegetables. There is also a shopping cart. Unity 5.3.5f1 (https://unity3d.com) and visual studio 2015 (Microsoft) were used to design and create the VRSTS.

The VRSTS included two tasks (task a and task $b$ ) and each task consisted of 4 different levels. Before each task started, there was a practice task which was used to bring all participants up to their best level of performance and the outcome of practice task was not included in statistical analysis.

In the practice task, participants become familiar with the procedures as follows:

The participants took the helmet to enter the VRSTS task;

A list of shopping goods appeared in the VR device and participant read the list and close it after remember the list;

The participants caught the good accordingly to put in the shopping cart in the virtual supermarket by using joysticks;

If the participant forgot the content of the list, he could press the button in the joysticks and the list would occur again.

The VR situation is presented as follows. (see Fig. 1)

Task a asked participants to find goods of certain kind and put them into the shopping carts, such as fruits, vegetables and drinks, while task b instructed participants to catch specific goods such as apples, tomatoes and cola. The number of goods ranged from 3 to 6 as the levels of task increased. While the good number increased, the working memory span needed is increased. As a result, different levels represented different difficulty levels.

When the participant caught all the goods of the list to the shopping cart, Computers automatically recorded the correct numbers, errors, and completion time of every task and calculated the accuracy. The accuracy is equal to the correct numbers divided by the number of goods. 
If the accuracy was less than 100 percent for the first time, the participant would enter the present level and repeat again. The second accuracy would be statistically analyzed.

Completion time and accuracy are major outcomes of the VR task, used for evaluating cognitive function. The experiment would not limit the completion time of the task so that every participant made the accuracy as high as possible.

\section{Cognitive Assessment}

Cognitive functioning was assessed by a trained psychiatrist using the MCCB. The MCCB includes 10 neurophysiologic tests clustered in 7 cognitive domains: speed of processing (SP), attention/vigilance $(\mathrm{AV})$, working memory (WM), verbal learning (VeL), visual learning (ViL), reasoning/problem solving (RPS), and social cognition (SC)[18]. Each domain score was standardized to a T score using the MCCB computer scoring program (Psychological Assessment Resources, Inc., version 2.1.1). Furthermore, the overall composite T score was calculated by averaging the standardized value of each test's $T$ score.

\section{Diagnosis Model And Verification}

We constructed a SVM-based cognitive impairment diagnosis model for patients with schizophrenia based on outcomes from 57 subjects (32 SZs and twenty five HCs), and outcomes from 18 additional subjects ( 9 SZs and nine HCs) were analyzed for verification of diagnostic effect.

\section{Statistical analysis}

The data are expressed as the mean \pm SD for continuous variables. T-tests were used to compare age, education year of the schizophrenia patients and healthy controls. The T scores of the MCCB and outcomes of VRSTS were analyzed by covariance analysis (covariates: years of education). Correlation of T scores of the MCCB and outcomes of VRSTS was analyzed by Pearson correlation. Statistical analyses were performed using SPSS version 19.0 (IBM, Chicago, IL, USA) for windows. As for verification of diagnostic model, DTREG (https://www.dtreg.com/) was used to test the diagnosis model.

\section{Results}

\section{Characteristics of SZs and HCs}

We recruited $35 \mathrm{SZs}$ and $25 \mathrm{HCs}$. Three out of the $35 \mathrm{SZs}$ were withdrawn because they could not finish the MCCB. Thirty-two SZs and $25 \mathrm{HCs}$ completed both the VRSTS and the MCCB. Five SZs and $4 \mathrm{HCs}$ felt dizzy during the VR tasks, but they finished all tasks because it was tolerable. No other uncomfortable feeling was reported both in SZs and HCs. The age of SZs ranged from 24 to 54 (mean age $=42.69, \mathrm{SD}=$ 9.01), while the age of HCs ranged from 28 to 51 (mean age = 38.84 SD = 5.56). Subject demographic and clinical characteristics are provided in Table 1. The duration of disease of SZs ranged from 12 to 384 months $(217.87 \pm 108.11)$. SZs received second generation antipsychotics (SGA) for treatment. There were no significant differences in age and gender between groups (all $P>0.05$ ). However, patients with 
SZ had fewer years of education $(10.50 \pm 3.14)$ than the healthy controls $(16.28 \pm 1.72)(P<0.001)$ (see Table 1).

Table 1

demographic and clinical characteristics of schizophrenic patients and healthy controls

\begin{tabular}{|lllll|}
\hline group & $\mathrm{SZ}(\mathbf{n}=\mathbf{3 2})$ & $\mathrm{HC}(\mathbf{n = 2 5 )}$ & $\mathrm{t} / \mathbf{\chi}^{2}$ & $\boldsymbol{p}$ \\
\hline Age (years) & $42.69 \pm 9.01$ & $38.84 \pm 5.56$ & $1.873^{\mathrm{a}}$ & 0.066 \\
\hline Gender (male/female) & $16 / 16$ & $8 / 17$ & $1.865^{\mathrm{b}}$ & 0.172 \\
\hline Education (years) & $10.50 \pm 3.14$ & $16.28 \pm 1.72$ & $-8.270^{\mathrm{a}}$ & $<0.001^{\star \star}$ \\
\hline Age of first onset (year) & $24.63 \pm 6.89$ & & & \\
\hline Duration (months) & $217.88 \pm 108.11$ & & & \\
\hline
\end{tabular}

\section{Cognitive function of patients with schizophrenia and healthy controls}

It took each participant approximately one hour to finish the MCCB. Covariance analysis (education) showed that SZs were significantly impaired in SP, AV, VeL, ViL, RPS, and SC compared to HCs (all P > 0.003) (see Table 2). 
Table 2

MCCB results of schizophrenic patients and healthy controls

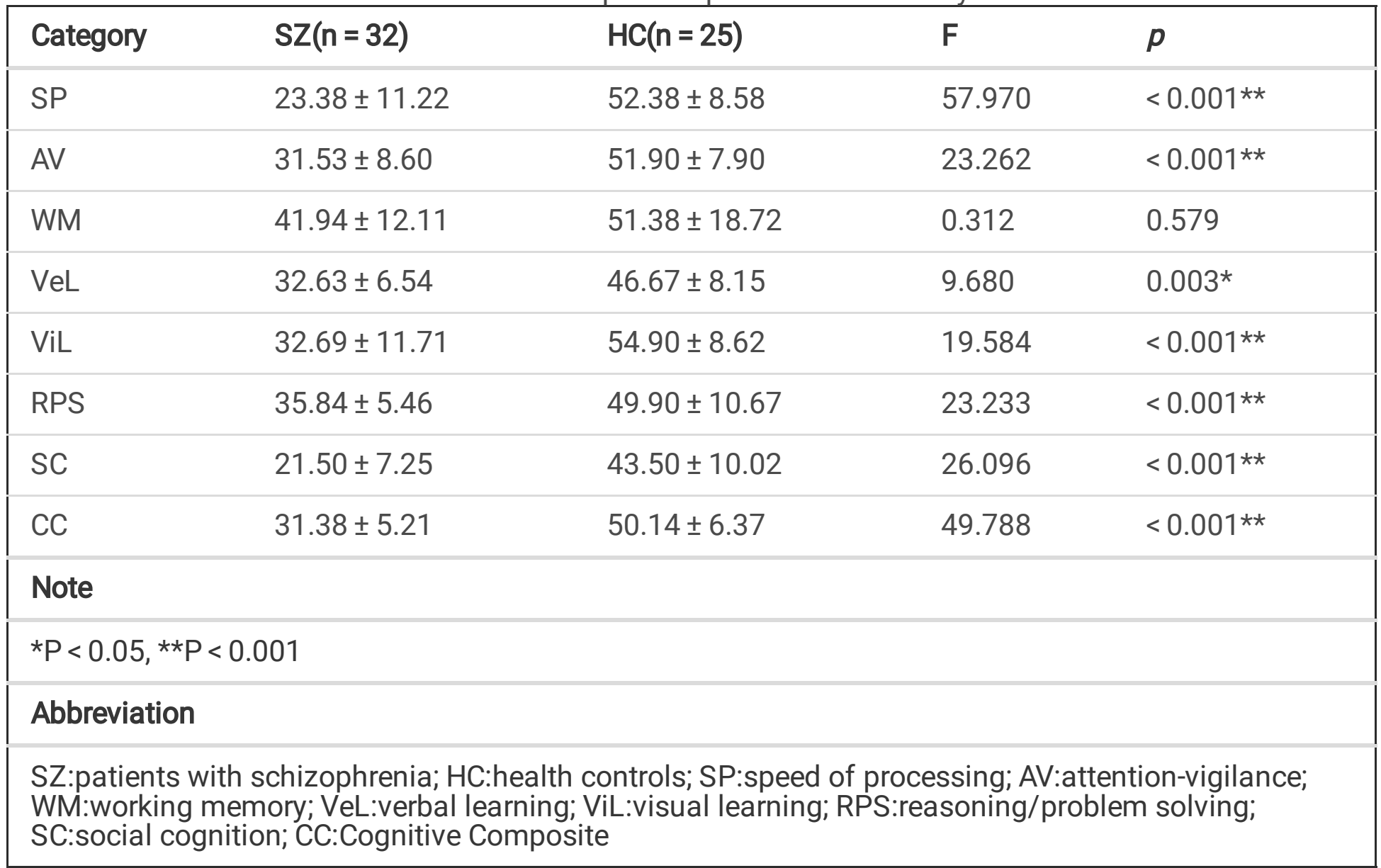

\section{Comparison of performance on the VRSTS between SZs and HCs}

The average total competition time of the VRSTS in SZs (1061 $\pm 427 \mathrm{~s})$ was significantly higher than that in HCs (389 \pm 226 s). Specifically, SZs spent significantly more time completing the different levels compared to HCs in Task a level 1, level 2 and level 3 as well as in Task b level 2 and level 3 (Bonferroni correction, all $\mathrm{P}<0.0125)$. However, the accuracy of every task showed no difference between patients with schizophrenia and healthy controls (see Table 3). 
Table 3

VRSTS outcome of patients with schizophrenia and healthy controls

\begin{tabular}{|c|c|c|c|c|}
\hline Tasks & & $S Z(n=32)$ & $\mathrm{HC}(n=25)$ & $p$ \\
\hline \multirow[t]{2}{*}{ Task a level 1} & Time to complete & $88.15 \pm 55.30$ & $31.75 \pm 26.40$ & $<0.001^{\star *}$ \\
\hline & Accuracy & $0.97 \pm 0.10$ & $0.96 \pm 0.11$ & 0.296 \\
\hline \multirow[t]{2}{*}{ Task a level 2} & Time to complete & $117.04 \pm 81.57$ & $29.91 \pm 11.62$ & $0.008^{*}$ \\
\hline & Accuracy & $0.95 \pm 0.12$ & $0.95 \pm 0.10$ & 0.481 \\
\hline \multirow[t]{2}{*}{ Task a level 3} & Time to complete & $139.81 \pm 76.51$ & $37.32 \pm 17.62$ & $0.006^{*}$ \\
\hline & Accuracy & $0.93 \pm 0.12$ & $0.95 \pm 0.09$ & 0.297 \\
\hline \multirow[t]{2}{*}{ Task a level 4} & Time to complete & $152.13 \pm 121.55$ & $44.10 \pm 18.21$ & 0.057 \\
\hline & Accuracy & $0.93 \pm 0.09$ & $0.96 \pm 0.09$ & 0.325 \\
\hline \multirow[t]{2}{*}{ Task b level 1} & Time to complete & $72.36 \pm 78.00$ & $43.26 \pm 50.90$ & 0.351 \\
\hline & Accuracy & $0.94 \pm 0.13$ & $0.89 \pm 0.19$ & 0.183 \\
\hline \multirow[t]{2}{*}{ Task b level 2} & Time to complete & $106.97 \pm 60.92$ & $44.21 \pm 34.56$ & $0.012^{\star}$ \\
\hline & Accuracy & $0.89 \pm 0.17$ & $0.86 \pm 0.19$ & 0.825 \\
\hline \multirow[t]{2}{*}{ Task b level 3} & Time to complete & $132.98 \pm 71.84$ & $54.69 \pm 41.16$ & $0.010 *$ \\
\hline & Accuracy & $0.88 \pm 0.15$ & $0.87 \pm 0.11$ & 0.950 \\
\hline \multirow[t]{2}{*}{ Task b level 4} & Time to complete & $179.83 \pm 117.25$ & $95.28 \pm 131.59$ & 0.211 \\
\hline & Accuracy & $0.90 \pm 0.13$ & $0.87 \pm 0.16$ & 0.132 \\
\hline \multicolumn{5}{|l|}{ Note } \\
\hline \multicolumn{5}{|c|}{ Bonferroni correction ${ }^{*} \mathrm{P}<0.0125,{ }^{\star \star *} \mathrm{P}<0.001$} \\
\hline \multicolumn{5}{|l|}{ Abbreviation } \\
\hline SZ:patients wi & schizophrenia; HC: & ealth controls & & \\
\hline
\end{tabular}

\section{Correlations of VRSTS outcome with the MCCB}

In this analysis, we selected the completion time of every task as well as the average completion time that discriminated between the HCs and schizophrenia patients and correlated them with the MCCB cognitive composite. These correlations are presented in Table 4 and Fig. 2. As shown in the Table 4, the average completion time of task $a$, task $b$ and many tasks were significantly negatively correlated with the MCCB cognitive composite.(Bonferroni correction, all $\mathrm{P}<0.0125)$. 
Table 4

Pearson correlations between VRSTS and MCCB in patients with schizophrenia

\begin{tabular}{|lll|}
\hline Time to complete & MCCB Cognitive Composite & $\mathbf{p}$ \\
\hline Task a level 1 & -0.487 & $<0.001^{\star \star}$ \\
\hline Task a level 2 & -0.604 & $<0.001^{\star \star}$ \\
\hline Task a level 3 & -0.723 & $<0.001^{\star \star}$ \\
\hline Task a level 4 & -0.493 & $<0.001^{\star \star}$ \\
Average of task a & -0.699 & $<0.001^{\star \star}$ \\
\hline Task b level 1 & -0.310 & 0.032 \\
\hline Task b level 2 & -0.468 & $0.001^{\star}$ \\
\hline Task b level 3 & -0.382 & $0.007^{\star}$ \\
\hline Task b level 4 & -0.12 & 0.415 \\
Average of task b & -0.441 & $0.002^{\star}$ \\
Average of all & -0.625 & $<0.001^{\star \star}$ \\
\hline
\end{tabular}

\section{Using the VRSTS to identify cognitive impairment}

Our results showed the cognitive impairment diagnosis model correctly classified SZs and HCs by cognitive impairment. The competition time of VRSTS in classifying cognitive impairment was $92.98 \%$ accuracy, $90.63 \%$ sensitivity, and $96 \%$ specificity. The area under the ROC curve was 0.9325 (see Fig. 3). And verification based on another 18 subjects showed $88.89 \%$ accuracy, $88.89 \%$ sensitivity, $88.89 \%$ specificity, $88.89 \%$ positive predictive value and $88.89 \%$ negative predictive value.

\section{Discussion}

The present study built a virtual situation called VRSTS, simulating a real supermarket to assess cognitive function and explored the validity and safety of VR technology used in the cognitive function of Han Chinese patients with schizophrenia. It took SZs nearly 18 minutes and HCs 6 minutes to complete the VRSTS, which is less than the completion time of the MCCB.

The VRSTS was divided into two tasks and each tasks consisted of 4 levels reflecting increasing working memory span. Task a was used to evaluate the processing of information related to certain categories, while task $b$ was used to assess the processing of specific information. We designed different levels for each task, such as task a level 1 and level 2. The number of goods needed to catch ranged from 3 to 6 as 
the levels of task increased. The outcome was measured by two variables: accuracy of performance and the time to complete the task. Because the accuracy of tasks showed no difference between SZs and $\mathrm{HCs}$, it is meaningful to compare the completion time of the two groups.

Our results showed that the completion time of task a and task b of SZs was both significantly higher than that of HCs, which was likely due to the cognitive impairment of patients with schizophrenia. These findings coincide with some studies. Thirty-three SZs and $39 \mathrm{HCs}$ performed ten trials of a virtual radial arm maze task, and the results showed that SZs took more time to finish the task[19]. Other studies also showed that SZs performed worse than HCs in the Virtual Action Planning-Supermarket (VAP-S) study and a Virtual Reality Functional Skills Assessment (VRFAS)[20, 21]. Furthermore, using virtual reality could assess the severity of theory of mind (ToM) impairment of SZs[22].

In addition, there is a significantly negative correlation between the average completion time of each task and the MCCB composite score, which support our hypothesis that the VRSTS outcome may reflect the cognitive impairment of patients with schizophrenia in the remission stage.

Furthermore, our results showed that the VRSTS could distinguish patients with schizophrenia and healthy controls with high accuracy (88.89\%), sensitivity (88.89\%) and specificity (88.89\%). This means that patients with schizophrenia can be separated from healthy people based on their performance in the VRSTS. This result is consistent with a study of a virtual reality prospective memory test demonstrating that the VR test could examine prospective memory deficits in patients with schizophrenia with high sensitivity (92.9\%) and specificity (75\%) [14].

In summary, the present study demonstrates that virtual reality technology, such as the VRSTS, is a timesaving, efficient and attractive method to evaluate cognitive function. It builds a virtual situation applicable to Han Chinese people to evaluate cognitive function and its outcome has a significant correlation with the MCCB. Moreover, it could precisely distinguish patients with schizophrenia and healthy people by cognitive impairment, indicating that it might be a new adjunctive examination to evaluate cognitive impairment in patients with schizophrenia in the remission stage, especially in those of Han Chinese descent.

This study had some limitations. Firstly, the sample size of participants is small. Secondly, we did not ask participants to assess their preference for MCCB vs VRSTS. So it lacks the feedback of participants after they finish VRSTS; as a result, it is difficult to rate the satisfaction and pleasantness the participants experienced. Moreover, the SCs received different types of second-generation antipsychotics, which may interfere with the patients' cognitive function. Furthermore, the two groups were not education matched, it may influence their performance in virtual supermarket. So we used covariance analysis (covariates: years of education) to compare the outcome between patients with schizophrenia and healthy controls. Last but not the least, VRSTS is an assessment to evaluate cognitive function deficit but as it simulated a daily activity, choosing goods in a supermarket, the task involves many domains of cognitive function such as attention, verbal learning, visual learning and working memory span. As a result it was not closely related with a specific cognitive domain except working memory span. 


\section{Conclusions}

The VRSTS is a highly sensitive measure of cognitive function associated with the MCCB test. These results provide support for VR technology in the assessment of cognitive function in schizophrenia among Han Chinese patients.

\section{Abbreviations}

SZ: patients with schizophrenia

HC: health controls

\section{Declarations}

\section{Ethical Approval and Consent to participate}

All participants provided informed consent prior to participation in the study.

The study was approved by the ethics committee of the First Affiliated Hospital of the Medical School of Zhejiang University in accordance with the Declaration of Helsinki

\section{Consent for publication}

All participants provided consent for publication of their data prior to participation in the study.

\section{Availability of supporting data}

The datasets used and/or analysed during the current study are available from the corresponding author on reasonable request.

\section{Competing interests}

The authors declare that they have no competing interests

\section{Funding}

This study was supported by two grants 2016YFC1307005 and 2016YFC1306900 from the National Key Research and Development Program of China, a grant LGF18H090003 of Basic Public Welfare Research projects in Zhejiang province, a grant 2015C03054 of major subject of Zhejiang Province and a grant 2016 C37076 from the Zhejiang provincial science and Technology Department.

\section{Authors' contributions}

YX and $\mathrm{MH}$ contributed to the study design and concept and wrote the protocol. SL managed the literature searches, statistical analysis and manuscript writing. Bin Sun contributed to data acquisition. 
HL and WX helped with the statistical analysis. JJ, FP, JH, JC and YC assisted with the experiment. All authors were involved in the preparation and review of the manuscript and approved the final version to be submitted.

\section{Acknowledgements}

We would like to thank the patients for entering the experiment and for their time and effort devoted to the consent process and interviews. We would also like to thank the staff in the Ningbo Psychiatric Hospital for their assistance.

\section{References}

1. Owen MJ, Sawa A, Mortensen PB: Schizophrenia. Lancet 2016, 388(10039):86-97.

2. Joyce EM, Roiser JP: Cognitive heterogeneity in schizophrenia. Curr Opin Psychiatry 2007, 20(3):268272.

3. Fioravanti M, Bianchi V, Cinti ME: Cognitive deficits in schizophrenia: an updated metanalysis of the scientific evidence. BMC Psychiatry 2012, 12:64.

4. Shi C, Kang L, Yao S, Ma Y, Li T, Liang Y, Cheng Z, Xu Y, Shi J, Xu X et al: The MATRICS Consensus Cognitive Battery (MCCB): Co-norming and standardization in China. Schizophr Res 2015, 169(13):109-115.

5. Nuechterlein KH, Green MF, Kern RS, Baade LE, Barch DM, Cohen JD, Essock S, Fenton WS, Frese FJ, 3rd, Gold JM et al: The MATRICS Consensus Cognitive Battery, part 1: test selection, reliability, and validity. Am J Psychiatry 2008, 165(2):203-213.

6. McCleery A, Ventura J, Kern RS, Subotnik KL, Gretchen-Doorly D, Green MF, Hellemann GS, Nuechterlein KH: Cognitive functioning in first-episode schizophrenia: MATRICS Consensus Cognitive Battery (MCCB) Profile of Impairment. Schizophr Res 2014, 157(1-3):33-39.

7. Cao A, Shen T, Li H, Wu C, McCabe M, Mellor D, Byrne L, Zhang J, Huang J, Peng D et al: Dysfunction of Cognition Patterns Measured by MATRICS Consensus Cognitive Battery (MCCB) among First Episode Schizophrenia Patients and Their Biological Parents. Shanghai Arch Psychiatry 2017, 29(3):154-160.

8. Freeman D, Reeve S, Robinson A, Ehlers A, Clark D, Spanlang B, Slater M: Virtual reality in the assessment, understanding, and treatment of mental health disorders. Psychol Med 2017, 47(14):2393-2400.

9. Mohammadi A, Hesami E, Kargar M, Shams J: Detecting allocentric and egocentric navigation deficits in patients with schizophrenia and bipolar disorder using virtual reality. Neuropsychol Rehabil 2018, 28(3):398-415.

10. Sorkin A, Weinshall D, Modai I, Peled A: Improving the accuracy of the diagnosis of schizophrenia by means of virtual reality. Am J Psychiatry 2006, 163(3):512-520. 
11. Kurtz MM, Baker E, Pearlson GD, Astur RS: A virtual reality apartment as a measure of medication management skills in patients with schizophrenia: a pilot study. Schizophr Bull 2007, 33(5):11621170.

12. Baker EK, Kurtz MM, Astur RS: Virtual reality assessment of medication compliance in patients with schizophrenia. Cyberpsychol Behav 2006, 9(2):224-229.

13. Dyck M, Winbeck M, Leiberg S, Chen Y, Mathiak K: Virtual faces as a tool to study emotion recognition deficits in schizophrenia. Psychiatry Res 2010, 179(3):247-252.

14. Man DWK, Ganesan B, Yip CCK, Lee COP, Tsang SYL, Yu PWP, Young JGE, Shum DHK: Validation of the virtual-reality prospective memory test (Hong Kong Chinese version) for individuals with firstepisode schizophrenia. Neuropsychol Rehabil 2018, 28(7):1197-1210.

15. Keefe RSE, Davis VG, Atkins AS, Vaughan A, Patterson T, Narasimhan M, Harvey PD: Validation of a Computerized test of Functional Capacity. Schizophr Res 2016, 175(1-3):90-96.

16. Ruse SA, Harvey PD, Davis VG, Atkins AS, Fox KH, Keefe RS: Virtual Reality Functional Capacity Assessment In Schizophrenia: Preliminary Data Regarding Feasibility and Correlations with Cognitive and Functional Capacity Performance. Schizophr Res Cogn 2014, 1(1):e21-e26.

17. Andreasen NC, Carpenter WT, Jr., Kane JM, Lasser RA, Marder SR, Weinberger DR: Remission in schizophrenia: proposed criteria and rationale for consensus. The American journal of psychiatry 2005, 162(3):441-449.

18. Sui J, Pearlson GD, Du Y, Yu Q, Jones TR, Chen J, Jiang T, Bustillo J, Calhoun VD: In search of multimodal neuroimaging biomarkers of cognitive deficits in schizophrenia. Biol Psychiatry 2015, 78(11):794-804.

19. Spieker EA, Astur RS, West JT, Griego JA, Rowland LM: Spatial memory deficits in a virtual reality eight-arm radial maze in schizophrenia. Schizophr Res 2012, 135(1-3):84-89.

20. Park KM, Ku J, Park IH, Park JY, Kim SI, Kim JJ: Improvement in social competence in patients with schizophrenia: a pilot study using a performance-based measure using virtual reality. Hum Psychopharmacol 2009, 24(8):619-627.

21. Josman $N$, Schenirderman $A E$, Klinger $E$, Shevil $E$ : Using virtual reality to evaluate executive functioning among persons with schizophrenia: a validity study. Schizophr Res 2009, 115(2-3):270277.

22. Canty AL, Neumann DL, Shum DHK: Using virtual reality to assess theory of mind subprocesses and error types in early and chronic schizophrenia. Schizophr Res Cogn 2017, 10:15-19.

\section{Figures}




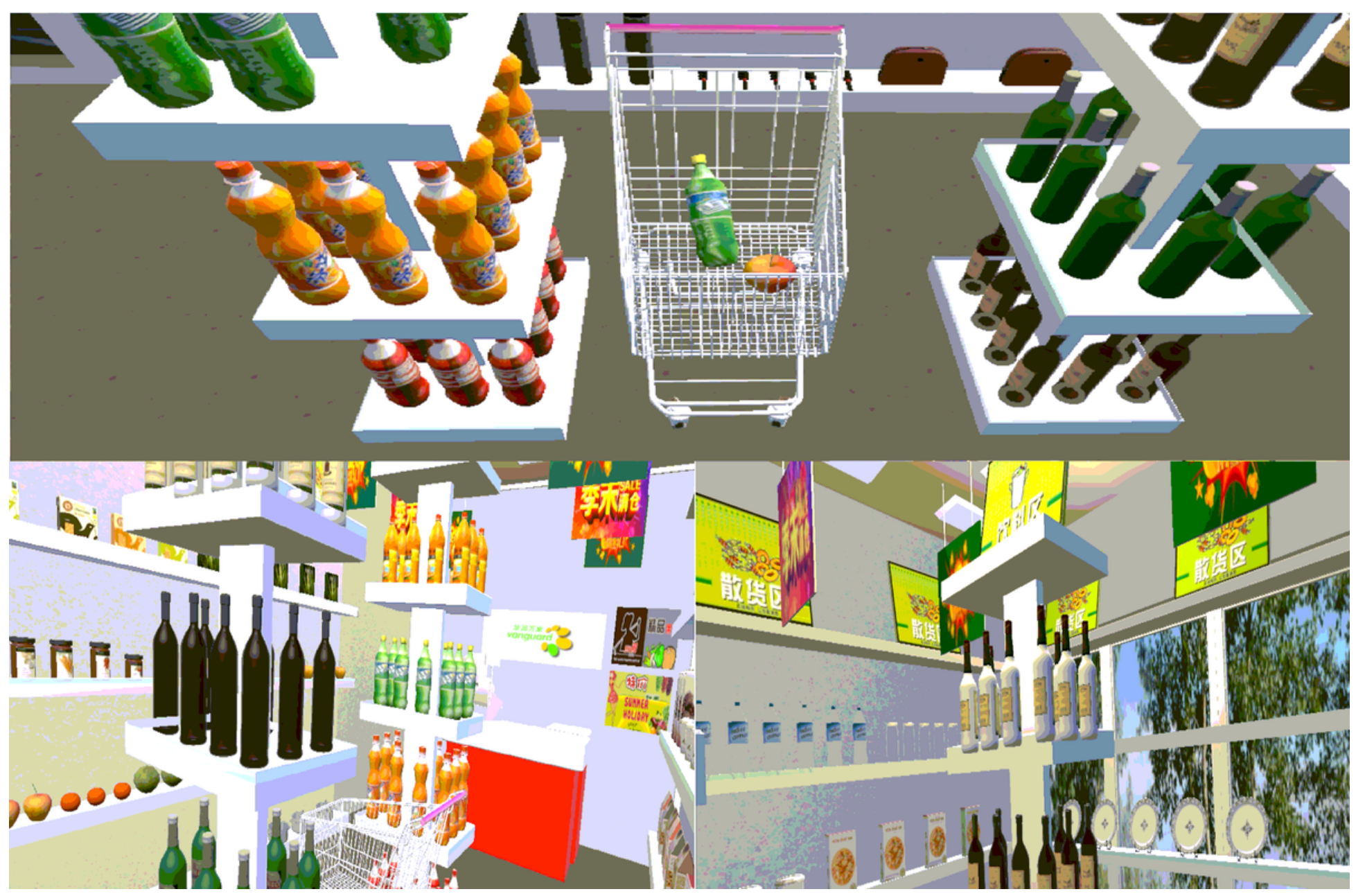

Figure 1

Screenshots showing different views within the virtual reality supermarket. The shopping cart (upper) and the goods (lower). 

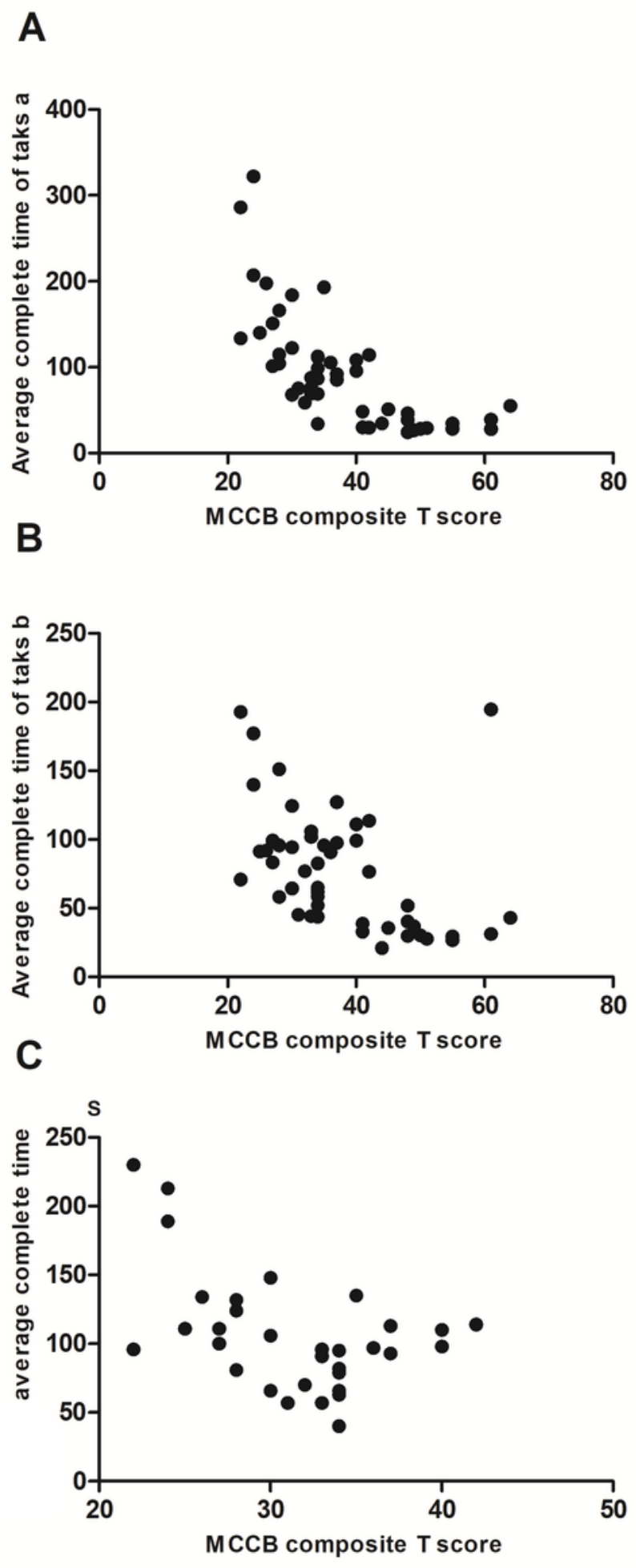

Figure 2

Scatter plots showing the correlation between the complete time of the VRSTS and the MCCB T scores. Completion time of Task a and MCCB T scores (A), completion time of Task b and MCCB T scores (B) average completion time of VRSTS and MCCB T scores (C). 


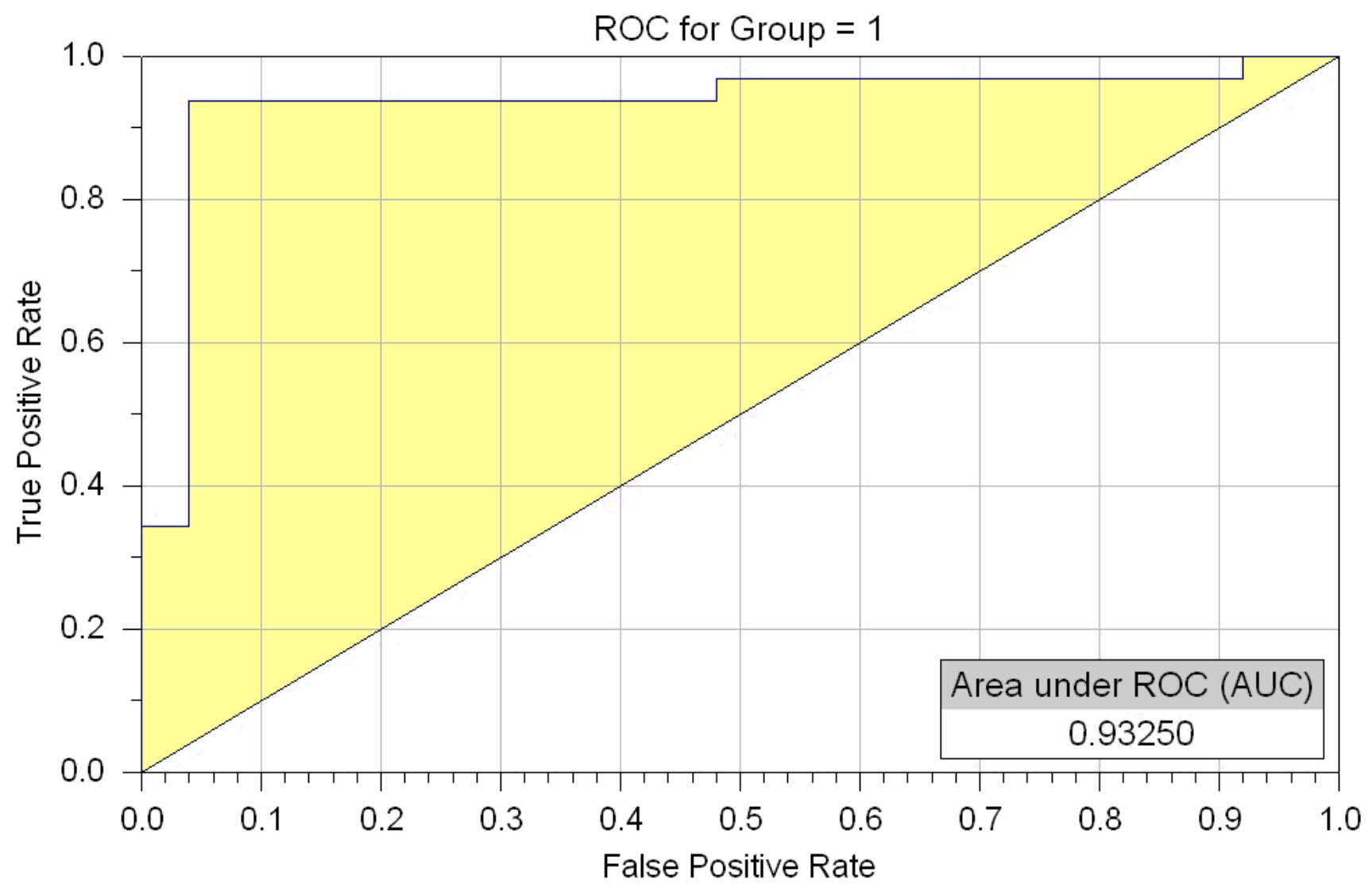

Figure 3

ROC curve to distinguish HCs and SZs. 